\title{
Primitive Mediastinal Hydatid Cyst
}

\author{
K Chaanoun*, H Benjelloun, N Zaghba, N Yassine
}

Department of Respiratory Disease, Chu Ibn Rochd, 1, Rue des Hôpitaux, Casablanca, Morocco

DOI: $\underline{10.36347 / \text { sjmcr.2020.v08i07.019 }}$

| Received: 14.07.2020 | Accepted: 22.07.2020 | Published: 28.07.2020

*Corresponding author: K Chaanoun

Abstract

Case Report

Mediastinal localization of the hydatid cyst is very rare even in endemic areas. We report the observation of a 24 year old patient of rural origin, having contact with dogs. Admitted to the respiratory diseases department for a huge multivesicular mediastinal mass. A thoracotomy is performed revealing a mediastinal hydatid cyst.

Keywords: Hydatid Cyst; mediastinum; echinococcosis.

Copyright @ 2020: This is an open-access article distributed under the terms of the Creative Commons Attribution license which permits unrestricted use, distribution, and reproduction in any medium for non-commercial use (NonCommercial, or CC-BY-NC) provided the original author and source are credited.

\section{INTRODUCTION}

Hydatidosis is a parasitosis caused by the development in humans of the larval form of Echinococcus granulosus. It is rampant endemic in countries experiencing significant development of sheep farming and having a humid climate favoring the survival of parasitic eggs, especially the countries around the Mediterranean, the Middle East and Latin America. Mediastinal localization of thoracic hydatidosis remains extremely rare. It represents 0.1 to $4 \%$ of all locations $[1,2]$.

\section{ObSERVATION}

This is a 24 year old patient of rural origin, who consults for chest pain and left flank associated with dyspnea of effort evolving for six months in a context of apyrexia and conservation of the state general. The clinical examination at admission is normal. The chest radiograph shows an opacity of water tone well limited in the retro-cardiac (Figure-1). The thoracic computed tomography objective a voluminous multi-vesicular cystic lesion with polycyclic contours measuring $17 \times 11 \times 14 \mathrm{~cm}$, involving the middle and posterior mediastinum, containing thick partitions and enhancing after injection of contrast product at the periphery. This mass pushes back the heart and the other elements of the mediastinum without obvious invasion (Figure-2). The CT aspect is rather in favor of a cystic lymphangioma. The hydatid serology is positive. The remainder of the assessment is without particularity in particular no extra-thoracic localizations. Diagnostic and therapeutic surgery is indicated. The intervention consisted of a posterolateral thoracotomy, which reveals a mediastinal hydatid cyst for which a peri-cystectomy is performed, the operative suites were simple. The patient was put on anti parasitic treatment for six months postoperatively. After a twoyear follow-up, the patient is asymptomatic and the radiological control is not objectified of hydatid recurrence.

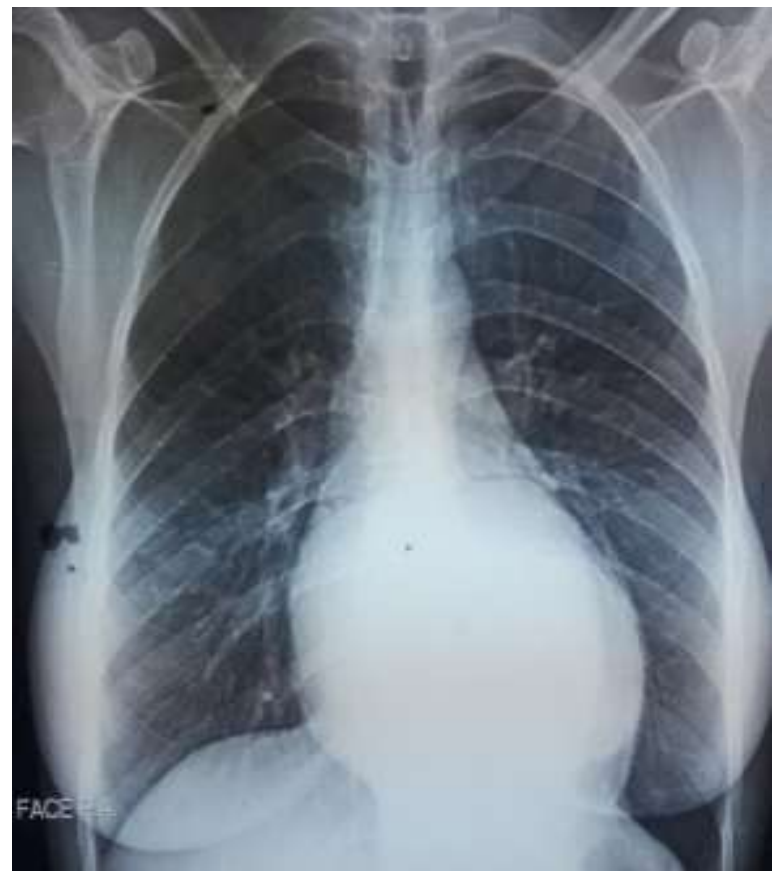

Fig-1: Chest x-ray showing a retro-cardiac opacity of water density 


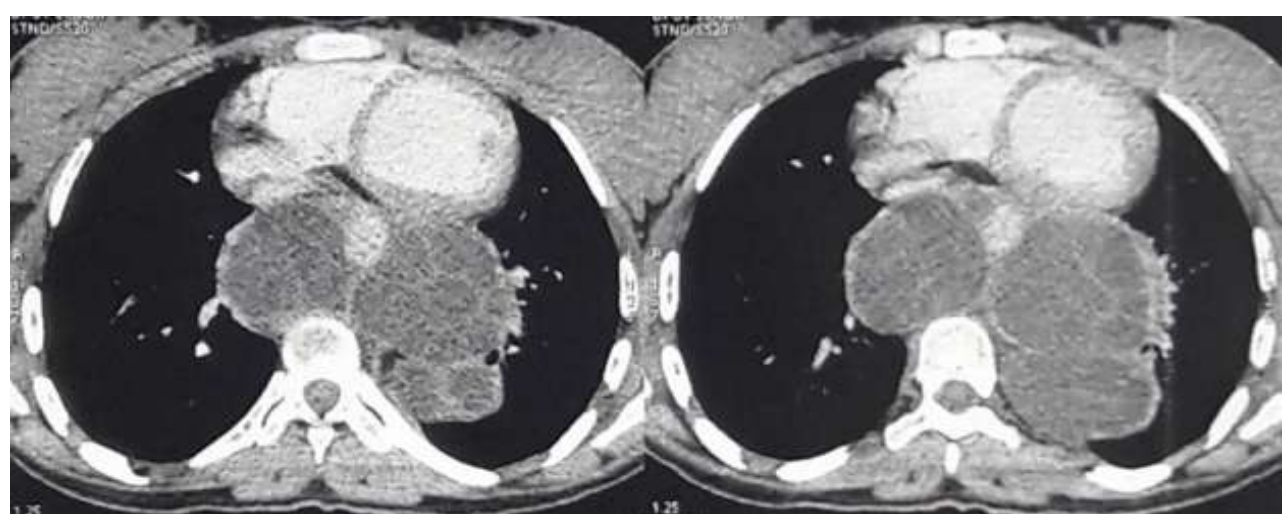

Fig-2: Thoracic CT scan in axial section, showing an enormous mediastinal mass containing septas and arriving in contact with the cardiac cavities and the spine

\section{DISCUSSION}

Mediastinal localization of the hydatid cyst is rare even in an endemic country like Morocco. The pathogenesis of this localization is still poorly defined. The parasite can reach the mediastinum either hematogenously, via the systemic circulation after passing through the hepatic and pulmonary filters, or via the lymphatic routes. A third mechanism has been described, that of trans-diaphragmatic dissemination from a hydatid cyst of the hepatic dome [3].

The mediastinal hydatid cyst is most often unique, sometimes multiple secondary to a spontaneous, traumatic or surgical rupture of a simple hydatid cyst.

The clinical symptomatology is not very specific, dominated by signs of irritation or compression of the neighboring organs. All localizations in the mediastinum are possible with a predilection for the posterior mediastinum. In a review of the literature reported by Rakower and Milwidsky: $55 \%$ of cysts sit at the level of the para-vertebral groove, $36 \%$ at the level of the anterior mediastinum, and less than $8 \%$ at the level of the visceral mediastinal compartment [4].

The location in the posterior mediastinum is the cause of pain and neurological signs secondary to vertebral or costal bone erosion. Localization in the anterior mediastinum may be responsible for a superior vena cava syndrome or dyspnea due to tracheal compression. At the level of the mediastinum the hydatid cyst can cause a hemorrhage by vascular erosion $[4,5]$.

In our patient the hydatid cyst is voluminous occupying the middle and posterior mediastinum with intimate contact with the large vessels and the heart, hence the need for urgent surgical excision, to avoid rupture in the aorta, in the cavities heart or in the mediastinum.

Imaging plays an essential role in the positive diagnosis and assessment of the extension of the hydatid cyst. The chest x-ray can guide the diagnosis by displaying a round mediastinal opacity of water tone. It can rarely objectify calcifications of the wall of the cyst. Thoracic ultrasound remains an essential examination if the lesion is accessible, by displaying the proliferous membrane doubling inside the pericyst, this sign is pathognomonic of the hydatid cyst [3]. The computed tomography shows a mass of fluid density, well limited, not increasing after injection of the contrast product. The hydatid cyst may contain fine partitions testifying to its multivesicular character or characteristic peripheral calcifications $[3,6]$. It also makes it possible to specify the relationships with the elements of the mediastinum.

Thoracic magnetic resonance imaging (MRI) makes it possible to specify the relationships with vascular structures and also remains useful in the diagnosis of cardiac localization which must be sought [5].

The presence of a mediastinal cystic mass suggests other diagnoses such as cystic lymphangioma, which remains more common in children and occurs mainly at the cervical level with mediastinal extension. The bronchogenic cyst which is spontaneously hyperdense and whose thick wall can calcify. The enteric cyst of the posterior seat in contact with the esophagus, its density is variable depending on its content and its wall is relatively thick. This cyst is most often associated with vertebral malformations. And finally the pleuropericardial cyst which is most often asymptomatic, triangular in appearance and pointed at the level of the non-declining part $[6,7]$.

In our patient, the contrast enhancement by the intra-cystic septas and at the periphery of the cystic mass complicated the situation by first evoking a cystic lymphangioma and it is only in intraoperative that the diagnosis of cyst hydatid has been worn.

Hydatid serology remains a fundamental examination in the positive diagnosis of hydatid cyst. Puncture biopsy is contraindicated during hydatidosis due to the potential risk of anaphylactic shock. 
The treatment of the mediastinal hydatid cyst is essentially surgical. Total peri-cystectomy removing the closed cyst is the method of choice. Subtotal perikystectomy or resection of the protruding dome is reserved for cases where the cyst develops in contact with the vascular-nervous axes. The evolution is favorable in the majority of the series of the literature.

\section{Conclusion}

In an endemic country like Morocco, the diagnosis of the mediastinal hydatid cyst must be evoked before any mediastinal mass. Imaging plays an essential role in the positive diagnosis of the hydatid cyst. Sometimes the doubt persists, especially in the absence of other localizations and it is only in intraoperative that the diagnosis is confirmed.

\section{REFERENCE}

1. Traibi A, Atoini F, Zidane A, Arsalane A, Kabiri el H. Mediastinal hydatid cyst. J Chin Med Assoc. 2010 Jan;73(1):3-7.
2. Thameur H, Chenik S, Abdelmoulah S, Bey M, Hachicha S, Chemingui M. Thoracic hydatidosis. A review of 1619 cases. Rev Pneumol Clin. 2000 Feb;56(1):7-15.

3. Karnak I, Ciftci AO, Tanyel FC. Hydatid cyst: an unusual etiology for a cystic lesion of the posterior mediastinum. J Pediatr Surg. 1998 May; 33(5):759-60.

4. Rakower J, Milwidsky H. Primary mediastinal echinococcosis. Am J Med. 1960 Jul; 29:73-83.

5. Della Sala M, Sessa M, Stana C, Carallo OF, Vescio G, Triggiani E. The magnetic resonance pictures of thoracic echinococcosis. A report of two cases. Radiol Med (Torino). 1995; 8(4):52832.

6. [6] Ben Mustapha A, Ben Hamadi F, Bouzaiani A, Ghyoula M, Hassine E, Ben Cheikh M. kyste hydatique thoracique extra pulmonaire. Med Trop (Mars). 1992 Oct-Dec; 52(4):399-405.

7. Akkari K, Chnitir S, Miled M, Mardassi A, Setthom A, Hachicha S, Chebbi M. Kyste hydatique cervico mediastinal à propos d'un cas. J Tun ORL. 2007; 19(1). 